\title{
Hosting Data Packages via drat: A Case Study with Hurricane Exposure Data
}

by G. Brooke Anderson and Dirk Eddelbuettel

\begin{abstract}
Data-only packages offer a way to provide extended functionality for other R users. However, such packages can be large enough to exceed the package size limit ( 5 megabytes) for the Comprehensive R Archive Network (CRAN). As an alternative, large data packages can be posted to additional repostiories beyond CRAN itself in a way that allows smaller code packages on CRAN to access and use the data. The drat package facilitates creation and use of such alternative repositories and makes it particularly simple to host them via GitHub. CRAN packages can draw on packages posted to drat repositories through the use of the 'Additonal_repositories' field in the DESCRIPTION file. This paper describes how $\mathrm{R}$ users can create a suite of coordinated packages, in which larger data packages are hosted in an alternative repository created with drat, while a smaller code package that interacts with this data is created that can be submitted to CRAN.
\end{abstract}

\section{Motivation}

"Big data", apart from being a buzzword, also accurately describes the current scale of many scientific data sets. While the R language and environment (R Core Team, 2017a) enables the creation, use, and sharing of data packages to support methodology or application packages, the size of these data packages can be very large. The Bioconductor project has addressed the potentially large size requirements of data packages through the use of Git Large File Storage, with the package contributor covering costs for extremely large data packages (over 1 gigabyte) (Bioconductor Core Team, 2017). The Bioconductor repository, though, is restricted to topic-specific packages related to bioinformatics. The Comprehensive $\mathrm{R}$ Archive Network (CRAN), which archives $\mathrm{R}$ packages on any topic, has a recommended size limit (reasonable for a widely-mirrored repository) of 5 megabytes (MB) for package data and documentation (R Core Team, 2017b). A twofold need therefore arises for package maintainers seeking to share large $\mathrm{R}$ data packages that are outside the scope of the Bioconductor project. First, there is a need to create and share such a data package for integration into and extensions of a given methodology or application package, and, second, there is a need to integrate use of such a package in a way that makes it seamlessly integrated with smaller CRAN packages that use the data package. Here, we outline one possible approach to satisfy these needs by creating a suite of coordinated packages, in which larger data packages are hosted outside of CRAN but can still be accessed by smaller code packages that are submitted to CRAN.

The problem of creating CRAN packages that interface with large datasets is not new, and various approaches have been taken in the past to allow for such an interface. For example, the NMMAPSlite package (currently available only from the CRAN archive) was built to allow users to interact with daily data on weather, air pollution, and mortality outcomes for over 100 US communities over 14 years (Peng and Dominici, 2008). To enable interaction with this large dataset through a CRAN package, the package maintainer posted the data on a server and included functions in the NMMAPSlite package to create an empty database on the user's computer that would be filled with community-specific datasets as the user queried different communities. This data interaction was enabled with the stash $\mathbb{R}$ package by the same maintainer (Eckel and Peng, 2012).

More recent packages similarly allow interaction between a web-hosted database and $\mathrm{R}$, in some cases for a database maintained by an outside entity. For example, the rnoaa package allows access to weather data posted by the National Oceanic and Atmospheric Administration (NOAA) (Chamberlain et al., 2016), while the tigris package allows access to spatial data from the United States Census Bureau (Walker and Rudis, 2016). Both packages are posted on CRAN and allow R users to work with a large collection of available online data by creating and sending HTTP requests from $\mathrm{R}$ using the conventions defined in the respective online databases' application program interfaces (APIs). This approach is a good one for data that is already available through an online database, especially if the database is outside the control of the R package maintainer or if the potential set of data is extremely large and it is unlikely that an R user would want to download all of it, since an API allows an R user to selectively download parts of the data. However, if the data is not already available through an online database, this approach would require the $\mathrm{R}$ package maintainer to create and maintain an online database, including covering the costs and managing the security of that database.

Another approach is to create a suite of packages, in which smaller ('code') packages are submitted to CRAN while larger ('data') packages are posted elsewhere. With this approach, the R user downloads all the data in the data package when he or she installs the data package, effectively 
caching this data locally so that data can be used in different $\mathrm{R}$ sessions without reloading or when the computer is offline. This can be a good approach in cases where the data is not otherwise available through an online database and when $\mathrm{R}$ users are likely to want to download the full set of data. In this case, this second approach offers several advantages, including: (1) the data can be documented through package helpfiles that are easily accessible from the R console; (2) if a user would like to delete all downloaded data from their computer, he or she can easily do so by removing the entire data package with remove. packages (as compared to other caching solutions, in which case the user might need to do some work to determine where a package cached data on his or her computer); (3) versioning can be used for the data package, which can improve reproducibility of research using the data (Gentleman et al., 2004); and (4) the data package can include the R scripts used to clean the original data (for example, in a 'data-raw' directory, with the directory's name excluded from the R package build through a listing in the '.Rbuildignore' file), which will make the package more reproducible for the package author and, if the full package directory is posted publicly (e.g., through a public GitHub repository), for other users.

The UScensus2000 suite of packages (currently available from the CRAN archive) used this approach to allow access to U.S. Census Bureau data from the 2000 Decennial Census (Almquist, 2010). This suite of packages included data at a more aggregated spatial level (e.g., state- and county-level data) through data packages submitted to CRAN, but included the largest dataset (block-level data) in an R package that was instead posted to the research lab's website (Almquist, 2010). A convenience function was included in one of the CRAN packages in the suite to facilitate installing this data package from the lab's website (Almquist, 2010).

This approach can be facilitated by posting the data package through an online package repository rather than a non-repository website. While support for repositories outside of CRAN, Bioconductor, and OmegaHat has existed within $\mathrm{R}$ for years, few users appear to have deployed this mechanism to host additional repositories. The drat package facilitates the creation and use of a user-controlled package repository. Once a package maintainer has created a repository, he or she can use it to host larger packages like a data package (and of course also any number of code packages). Use of a drat repository allows $\mathrm{R}$ users to install and update the data package using traditional $\mathrm{R}$ functions for managing packages (e.g., install . packages, update. packages) after the user has added the drat repository through a call to addRepo (Eddelbuettel et al., 2016). A drat repository can be published online through GitHub Pages, and GitHub repositories have a recommended maximum size of 1 GB, much larger than the size limit for a CRAN package, with a cap of $100 \mathrm{MB}$ on any single file (https://help.github.com/articles/what-is-my-disk-quota/). Even for data packages below the CRAN size limit, this approach to hosting data packages can help remove some of the burden of hosting and archiving large data packages from CRAN. Although the drat package is relatively new, some package maintainers are already taking this approach-for example, the grattan package facilitates research in $\mathrm{R}$ on Australian tax policy, with relevant data available through the large taxstats data package, posted in a drat repository.

When taking the approach of creating a suite of packages in which the smaller code package or packages are submitted to CRAN while larger data packages are hosted in drat repositories, it is necessary to add some infrastructure to the smaller code packages. CRAN policies do not allow the submission of a package with mandatory dependencies on packages hosted outside of a mainstream repository, which means that the smaller package could not be submitted to CRAN if the data package is included in the smaller package through 'Imports:' or 'Depends:'. The R package ecosystem offers a solution: a package maintainer can create a weaker relationship between code and data packages via a 'Suggests:', which makes the data package optional, rather than mandatory, as either 'Imports:' or 'Depends:' would. Being optional, one then has to condition any code in the code package that accesses data in the data package on whether that package is present on the user's system. This approach offers the possibility of posting a data package that is too large for CRAN on a non-mainstream respository, like a drat repository, while submitting a package that interacts with the data to CRAN.

This paper outlines in sufficient detail the steps one has to take to use drat to host a large data package for $\mathrm{R}$ and how to properly integrate conditional access to such an optional data package into a smaller code package. The packaging standard aimed for is the CRAN Repository Policy and the passing of 'R CMD check --as-cran'. Broadly, these steps are:

1. Create a drat repository;

2. Create the data package(s), build it, and post it in the drat repository; and

3. Create / alter the code package(s) to use the data package(s) in a way that complies with CRAN checks.

As a case study, we discuss and illustrate the interaction between the packages hurricaneexposure (Anderson et al., 2017b) and hurricaneexposuredata (Anderson et al., 2017a). The latter package contains data for eastern U.S. counties on rain, wind, and other hurricane exposures, covering all historical Atlantic-basin tropical storms over a few decades. The total size of the pack- 
age's source code is approximately $25 \mathrm{MB}$, easily exceeding CRAN's package size limit. This package includes only data, but the companion package, hurricaneexposure, provides functions to map and interact with this data. The hurricaneexposure package is of standard size and available from CRAN since the initial version 0.0.1, but to fully utilize all its capabilities requires access to the data in package hurricaneexposuredata. Here, we highlight specific elements of code in these packages that allow coordination between the two. The full code for both packages is available through GitHub repositories (https://github.com/geanders/hurricaneexposure and https: //github.com/geanders/hurricaneexposuredata); this article references code in version 0.0.2 of both packages.

\section{Posting a data package to a drat repository}

\section{Creating a drat repository}

A package maintainer must first create a drat repository if he or she wishes to host packages through one. Essentially, this repository is a way to store $\mathrm{R}$ packages such that it is easy for $\mathrm{R}$ users to download and update the packages; the repository can be shared, among other ways, through a GitHub-hosted website. Because a drat repository is controlled by the package maintainer, it allows increased flexibility to package maintainers compared to repositories like CRAN. A single drat repository can host multiple packages, so a maintainer likely only needs a single drat repository, regardless of how many packages he or she wishes to host on it.

A drat repository is essentially a network-accessible directory structure. The drat repository's directory must include index files (e.g., 'PACKAGES' and 'PACKAGES.gz'; Figure 1), which have metadata describing the packages available in the repository. The directory structure should also store files with the source code of one or more packages (e.g., 'hurricaneexposuredata_0.0.2.tar.gz') and can also include operating system-specific package code (e.g., 'hurricaneexposuredata_0.0.2.zip'). Multiple versions of a package can be included (e.g., 'hurricaneexposuredata_0.0.1.tar.gz' and 'hurricaneexposuredata_0.0.2.tar.gz' in Figure 1), allowing for archiving of old packages.

A user can create a drat directory with the required structure either by hand, via functions in the drat package, or by copying an existing drat repository (e.g., forking one on GitHub, like the original drat repository available at https://github. com/eddelbuettel/drat). While this directory can have any name, we suggest the user name the directory 'drat', as this allows the easy use of default variable names (which can of course be overridden as needed) for functions in the drat package, as shown in later code examples.

Second, for other users to be able to install packages from a drat repository, the repository must be available online. This can be easily achieved via the https protocol using GitHub's GitHub Pages, which allows GitHub users to create project webpages by posting content to an 'gh-pages' branch of the project's repository. (While there are now ways to publish content from a directory 'docs/' in the 'master' branch, functions in the drat package currently only support use of the older 'gh-pages' publishing option). Once this 'gh-pages' branch is pushed, the content in that branch will be available as part of the GitHub user's GitHub Pages website. For example, if the project is in the GitHub repository https://github.com/username/projectname, the content from the 'gh-pages' branch of that repository will be published at https://username.github.io/projectname.

Once this drat repository is created and published online through GitHub Pages, any source code or binaries for R packages within the repository can be installed and updated by $\mathrm{R}$ users through functions in the drat package. An $\mathrm{R}$ user can install a package from a drat repository by first adding the drat respository using the addRepo function from drat, with the appropriate GitHub username, and then using install . packages, as one would for a package on CRAN. The drat package documentation, including several vignettes, as well as supplementary webpages, have more detail on this process; see Eddelbuettel et al. (2016).

\section{Creating and building a data package}

The next step is to create an $\mathrm{R}$ data package that contains the large dataset; this data package will be posted in the drat repository to be accessible to other $\mathrm{R}$ users. In the case study example, this package is called hurricaneexposuredata and includes data on rain, wind, flood, and tornado exposures for all eastern US counties for Atlantic-basin tropical storms between 1988 and 2015. For full details on creating $\mathrm{R}$ packages, including data-only packages, see the canonical reference by R Core Team (2017b); another popular reference is Wickham (2015).

If a package is hosted in a drat repository rather than posted to CRAN, it does not have to pass all tests executed by 'R CMD check'. However, it is good practice to resolve as many ERRORS, WARNINGS, 


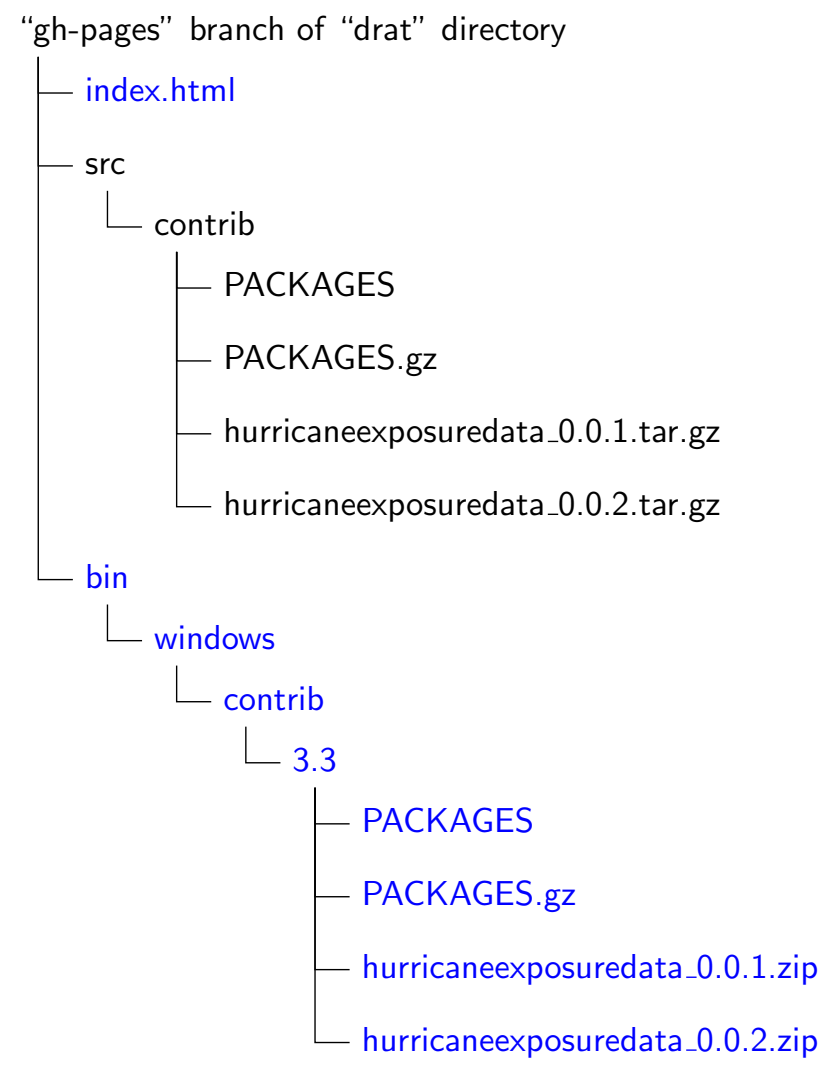

Figure 1: Example of the structure of the directory for a drat repository for a repository with two versions (0.0.1 and 0.0.2) of hurricaneexposuredata. Directories and files shown in black are required while those in blue are optional. This example drat depository has source code available for the hurricaneexposuredata, as well as the binaries for Windows (the binaries for Mac OS X could also be included but are not shown in this example), through the optional 'bin' subdirectory. The top-level 'index.html' file can be used to customize the appearance of the webpage a user would see at https: //username.github.io/drat. Functions from the drat package automate the insertion of compressed package files ('.tar.gz' for source code files, '.tgz' for Mac OS X binaries, and '.zip' for Windows binaries) within this directory structure. The 'PACKAGES' and 'PACKAGES.gz' files serve as index files, with metadata about packages available in the repository, and are created by drat: : insertPackage via a call to the R function tools: : write_PACKAGES. 
and NOTES from CRAN checks as possible for any R package that will be shared with other users, regardless of how it is shared. Several possibilities exist to build and check a package so these ERRORS, WARNINGS, and NOTES can be identified and resolved. The standard approach is to execute 'R CMD build' from one directory above the source directory (as discussed below), followed by 'R CMD check' with the resulting tar archive (e.g., hurricaneexposuredata. tar.gz) as first argument. The optional switch '--as-cran' is recommended in order to run a wider variety of tests. Other alternatives for checking the package are to use the check function from the devtools package (Wickham et al., 2016), the rcmdcheck function of the eponymous rcmdcheck package (Csárdi, 2016), the 'Check' button in the Build pane of the RStudio GUI, or the RStudio keyboard shortcut Ctrl-Shift-E. For a large data package, it is desirable to resolve all issues except the NOTE on the package size being large.

Once the code in the data package is finalized, the package can be posted in a drat repository to be shared with others. Packages are inserted into a drat repository as source code tarballs (e.g., '.tar.gz' files); if desired, package binaries for specific operating systems can also be inserted (e.g., '.zip' or '.tgz' files), but this is not required for the application described here. To build a package into a '.tar.gz' file, there are again several possible approaches. The most convenient one may be to build the package in a temporary directory created with the tempdir function, as this directory will be cleaned up when the current $\mathrm{R}$ session is closed. If the current working directory is the package directory, the package can be built to a temporary directory with the build function from the devtools package:

tmp <- tempdir()

devtools: : build (path $=\mathrm{tmp})$

While this function call assumes that the user is currently using the directory of the data package as the working directory, the pkg option of the build function can be used to run this call successfully from a different directory. If the build is successful, a '.tar.gz' file will be created containing the package's source code in the directory specified by the path option; this can be checked from $\mathrm{R}$ with the call list.files (tmp).

\section{Adding the data package to your drat repository}

Once the source code of the package has been built, the data package can be inserted into the drat repository using the insertPackage function from the drat package. This function identifies the package file type (e.g., '.tar.gz', '.zip', or '.tgz'), adds the package to the appropriate spot in the drat directory structure (Figure 1), and adds metadata on the package to the 'PACKAGES' and 'PACKAGES.gz' index files (created by the R function tools: : write_PACKAGES) in the appropriate subdirectory. Once this updated version of the drat repository is pushed to GitHub, the package will be available for other users to install from the repository.

For example, the following code can be used to add version 0.0 .2 of hurricaneexposuredata to the drat repository. This code assumes that the '.tar.gz' file for the package was built into a temporary directory with a path given by the R object tmp, as would be the case if the user built the package tarball using the code suggested in the previous subsection, and that the user is in the same R session as when the package was built (as any temporary directories created with tempdir are deleted when an $\mathrm{R}$ session is closed). Further, this code assumes that the user has the git2r package installed and has stored their drat directory within the parent directory /git; if this is not the case, the correct path to the drat directory should be specified in the repodir argument of insertPackage.

pkg_path <- file.path (tmp, "hurricaneexposuredata_0.0.2.tar.gz", sep = "/")

drat: : insertPackage (pkg_path, commit = TRUE)

As mentioned before, only material in the 'gh-pages' branch of the GitHub repository is published through GitHub Pages, so it is important that the package be inserted in that branch of the user's drat repository. The insertPackage function checks out that branch of the repository and so ensures that the package file is inserted in the correct branch. However, it is important that the user be sure to push that specific branch to GitHub to update the online repository. If unsure, the commit option can be left at its default value of FALSE, permitting an inspection of the repository followed by a possible manual commit.

For users who prefer working from the command line, an alternative pipeline for building the data package and inserting it into the drat repository is to run, from the command line:

R CMD build sourcedir/

dratInsert.r pkg_1.2.3.tar.gz

Note that this pipeline requires having the littler (Eddelbuettel and Horner, 2016) package installed, as well as the 'dratlnsert.r' helper script for that package. 
If desired, operating system-specific binaries of the data package can be built with tools like win-builder (http://win-builder.r-project.org/) and rhub (https://builder.r-hub.io) and then inserted into the drat repository. However, this step is not necessary, as ' $R$ CMD check --as-cran' will be satisfied for the code package as long as a source package is available for any suggested packages stored in repositories listed in 'Additional_repositories' (R Core Team, 2017b).

\section{Setting up a smaller code package to use the data package}

So far, the process described is the same one would use to create and add any $\mathrm{R}$ package to a drat repository. However, if a package maintainer would like to coordinate a code package that will be submitted to CRAN with a data package posted in a drat repository, it is necessary to add some infrastructure to the code package (in our example, hurricaneexposure, which has functions for exploring and mapping the data in hurricaneexposuredata). These additions ensure that the code package will pass CRAN checks and also appropriately load and access the data in the data package posted in the drat repository.

\section{Add infrastructure to the DESCRIPTION file}

First, two additions are needed and a third is suggested in the DESCRIPTION file (Figure 2) of the code package that will be submitted to CRAN:

1. We suggest the 'Description' field of the code package's 'DESCRIPTION' file be modified to let users know how to install the data package and how large it is (this tip is inspired by the grattan package; Parsonage et al. (2017)). Figure 2 (\#1) shows an example of this added information for the hurricaneexposure 'DESCRIPTION' file. For a CRAN package, this 'Description' field will be posted on the package's CRAN webpage, so this field offers an opportunity to inform users about the data package before they install the CRAN package. This addition is not required, but is particularly helpful in cases where the data package is very large, in which case it would take up a lot of room on a user's computer and take a long time to install and load.

2. The 'Suggests' field for the code package must specify the suggested dependency on the data package (Figure 2, \#2). Because the data package is in a non-mainstream repository, this dependency much be specified in the 'Suggests' field rather than the 'Depends' or 'Imports' field if the code package is to be submitted to CRAN. The 'Suggests' field allows version requirements, so if the code package requires either a minimum version or an exact version of the data package in the drat repository, this requirement can be included in this field.

3. The 'Additional_repositories' field of the code package must give the address of the drat repository that stores the data package (Figure 2,\#3). This field is necessary if a package depends on a package in a non-mainstream repository. Repositories listed here are checked by CRAN to confirm their availability (R Core Team, 2017b), but packages from these repositories are not installed prior to CRAN checks. This repository address should be listed using https: rather than http:.

\section{Customize behavior when the package is loaded}

When a package is installed, any packages listed as 'Imports' or 'Depends' in the package 'DESCRIPTION' file are guaranteed to be previously installed (R Core Team, 2017b); the same is not true for packages in 'Suggests'. It is therefore important that the maintainer of any package that suggests a data package from a drat repository take steps to ensure that the package does not fail if it is installed without the data package being previously installed. Such steps includes adding code that will be run when the package is loaded (described in this subsection), as well as ensuring that code in all functions, examples, vignettes, and tests be conditional on whether the data package installed if the code requires data from the data package (described in later subsections).

First, the code package should have code to check whether the data package is installed when the code package is loaded. This can be achieved through load hooks (.onLoad and .onAttach), saved to a file named, for example, 'zzz. $R$ ' in the ' $R$ ' directory of the code package (the name ' $z z z$. $R$ ' dates back to a time when $R$ required this; now any file name can be chosen). For a concrete example, such a 'zzz.R' file in the code package might look like (numbers in comments of this code are used within specific comments later in this section): 2 


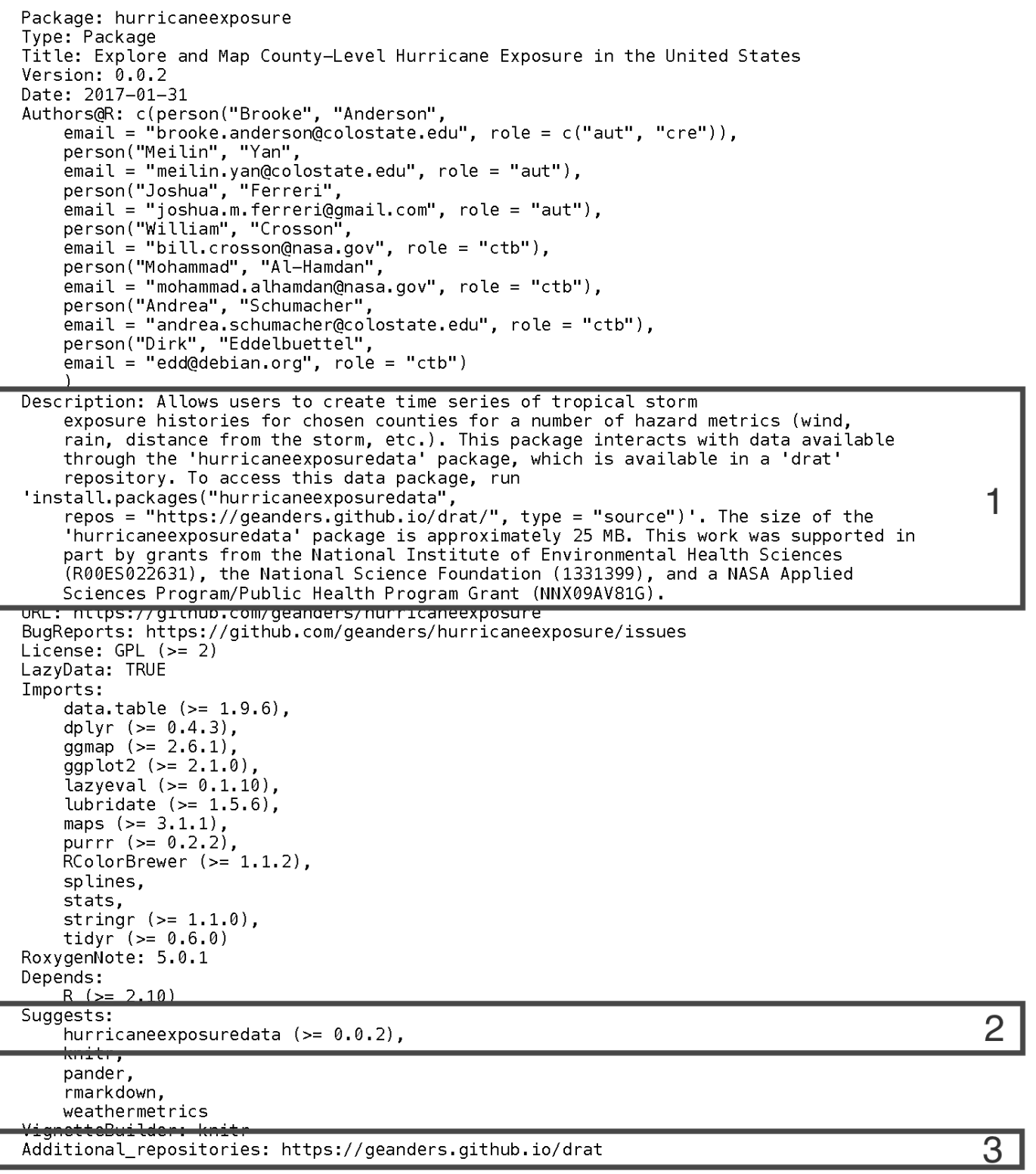

Figure 2: Example of the elements that should be added to the DESCRIPTION file of the code package planned to be submitted to CRAN to coordinate it with a data package posted to a drat repository, showing the DESCRIPTION file for hurricaneexposure. Elements are: (1) added details on installing the data package in the 'Description' field (suggested but not required); (2) suggested dependency on the data package in the 'Suggests' field; and (3) reference to the drat repository in the 'Additional_repositories' field. 


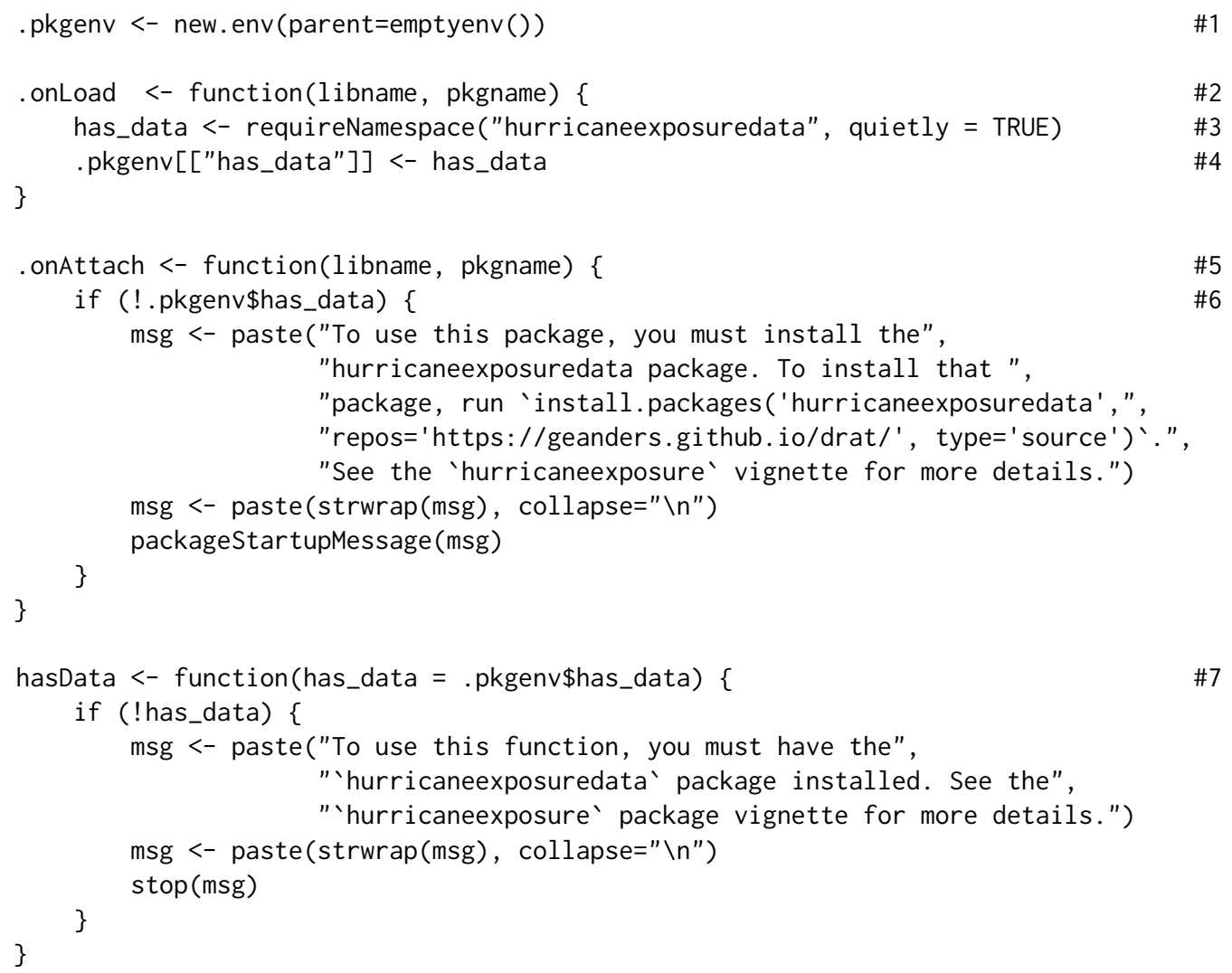

First, this 'zzz. $R$ ' file creates an environment called . pkgenv (\#1 in example code). This environment will be used to pass a Boolean variable indicating whether the data package is available (\#4) to other code in the package.

Next, this 'zzz.R' file defines two functions called . onLoad and . onAttach. Both functions have arguments libname and pkgname (\#2,\#5). The .onLoad() function, triggered when code from the package is first accessed, should use requireNamespace to test if the user has the data package available (\#3). This value is stored in the package environment (\#4). When the package is loaded and attached to the search path, the . onAttach function is called (\#5). This function references the stored Boolean value and uses this to print a start-up message (using the packageStartupMessage function, whose output can be suppressed via suppressPackageStartupMessages) for users who lack the data package (\#6).

Finally, any functions in the package that use data from the drat data package should check that the data package is available before running the rest of the code in the function. If the data package is not available, the function should stop with a useful error message. One way to achieve this is to define a function in an $\mathrm{R}$ script file in the package, like the hasData function defined in the example code above, that checks for availability of the data package and errors with a helpful message if that package is not installed (\#7). This function should then be added within all of the package's functions that require data from the data package.

\section{Condition code in the code package on availability of the data package}

Next, it is important to ensure that code in the vignettes, examples, and tests of the package will run without error on CRAN, even without the data package installed. When a package is submitted to CRAN, the user first creates a tarball of the package source on his or her own computer. In this local build, the vignette is rendered and the resulting PDF or HTML file is stored within the 'inst/doc' directory of the source code (R Core Team, 2017b). This is the version of the rendered vignette that is available to users when they install the package, and so the vignette is rendered using the packages and other resources available on the package maintainer's computer during the package build. However, CRAN runs initial checks on a package submission, and continues to run regular checks on posted packages, that include testing any executable code within the package vignette or any examples or tests in the source code that are not explicitly marked to not run on CRAN (e.g., with Idonttest\{\} within example code). CRAN does not install suggested packages from non-mainstream repositories before doing these checks. Therefore, code that requires data from a data package in a drat repository 
would cause errors in these tests unless it is conditioned to only run when the data package is available, as is recommended for any example or test code that uses suggested packages (R Core Team, 2017b).

Therefore, if the code package contains a vignette, the vignette must be coded so that its code will run without an error on systems that do not have the data package installed. This can be done by adding a code chunk to the start of the vignette. This code chunk will check if the data package is installed on the system. If it is, the vignette will be rendered as usual, and so it will be rendered correctly on the package maintainer's computer when the package is built for CRAN submission. However, if the optional data package is not installed, a message about installing the data package from the drat repository will be printed in the vignette, and all the following code chunks will be set to not be evaluated using the opts_chunk function from knitr (Xie, 2016).

The following code is an example of the code chunk that was added to the beginning of the vignette in the hurricaneexposure package, for which all code examples require the hurricaneexposuredata package:

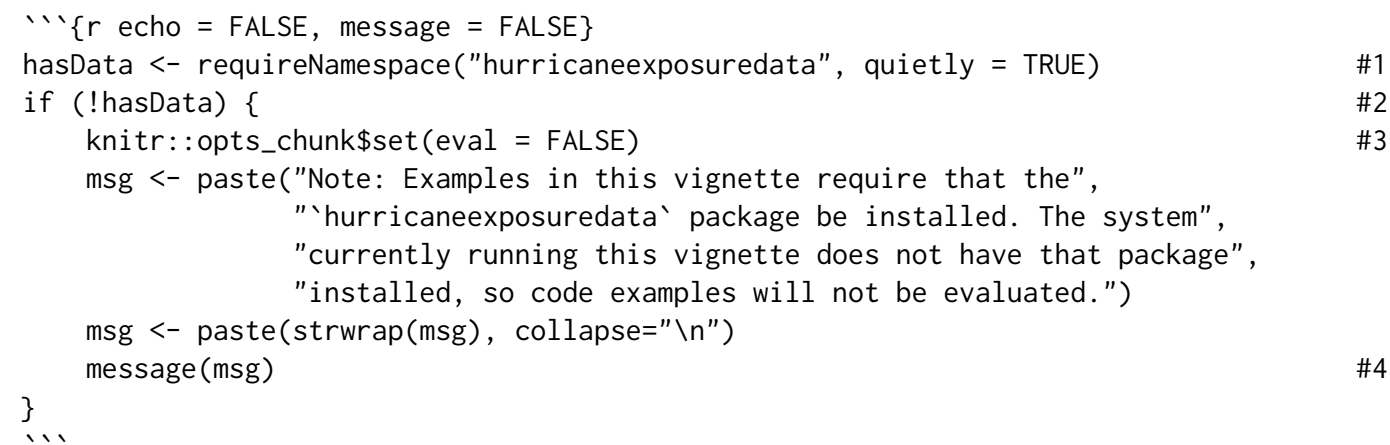

In this code, the function requireNamespace is used to check if hurricaneexposuredata is installed on the system (\#1). It is necessary to run this function in the vignette with the quietly = TRUE option; otherwise, this call will cause an error if hurricaneexposuredata is unavailable. If hurricaneexposuredata is not available, the result of this call is FALSE, in which case (\#2) the chunk option eval is set to FALSE for all following chunks in the vignette (\#3) and a message is printed in the vignette explaining why code chunks are not evaluated (\#4).

Similarly, the code for examples in help files of the CRAN package should be adjusted so they only run if the data package is available. It may also be helpful to users to include a commented message on why the example is wrapped in a conditional statement. For example, for a function that requires the data package the \examples \{\} field (or @examples tag if roxygen 2 is used) might look like:

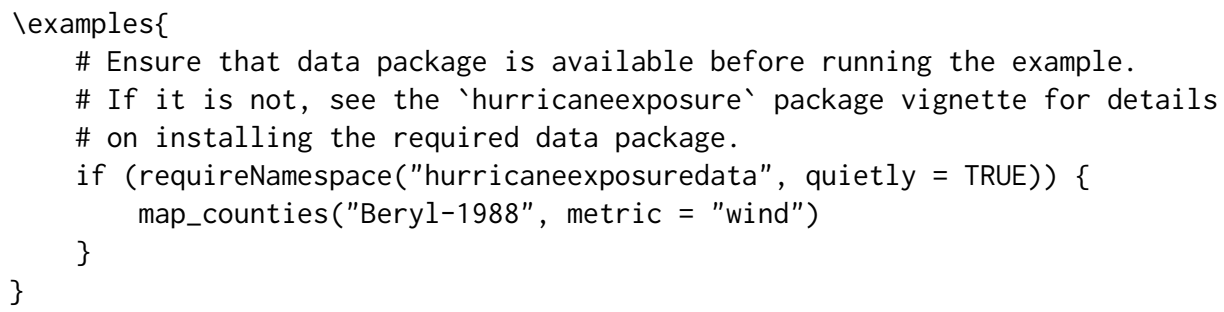

As alternatives, the code in the example could also either check the .pkgenv[["has_data"]] object created by code in the ' $z z z . R^{\prime}$ file in the conditional statement, or the package maintainer could create a helper function to in the ' $z z z$. $R$ ' file to use for conditional running of examples in the example code. However, the use of the requireNamespace call, as shown in the above code example, may be the most transparent for package users to understand when working through package examples. Code in package tests can similarly be conditioned to run if and only if the data package is available.

\section{Maintaining a suite of drat and CRAN R packages}

Once a suite of coordinated CRAN and drat packages have been created, there are a few considerations a package maintainer should keep in mind for maintaining the packages.

First, unlike a single package that combines data and code, a suite of packages will require thoughtful coordination between versions of packages in the suite, especially as the packages are updated. The 'Suggests' field allows the package maintainer to specify an exact version of the data package required by a code package (e.g., using ' $(==0.0 .1)^{\prime}$ with the data package name in the 
Suggests field) or a minimum version of the data package (e.g., '(>= 0.0 .1$\left.)^{\prime}\right)$. If the data package is expected to only change infrequently, the maintainer may want to use an exact version dependency in 'Suggests' and plan to submit an updated version of the code package to CRAN any time the data package is updated. However, CRAN policy recommends that package versions not be submitted more than once every one to two months. Therefore, if the data package will be updated more frequently, it may make more sense to use a minimum version dependency. However, the maintainer should be aware that, in this case, packages users may find that any changes in the structure of the data in the data package that breaks functions in older versions of the code package may cause errors, without notifying the user that the error can be resolved by updating the code package.

Second, while the maximum size of a GitHub repository is much larger than the recommended maximum size of an R package, there are still limits on GitHub repository size. If a package maintainer uses a drat repository to store multiple large data packages, with multiple versions of each, there may be some cases where the repository approaches or exceeds the maximum allowable GitHub repository size. In this case, the package maintainer may want to consider, when inserting new versions of the data package into the drat repository, changing the action option in the insertPackage function to free repository space by removing older versions of the package or packages. This, however, reduces reproducibility of research using the data package, as older versions would no longer be available through an archive.

Further, while the CRAN maintainers regularly run code from examples and vignettes within packages posted to CRAN, they do not install any suggested packages from non-mainstream repositories before doing this. Since much of the code in the examples and vignette are not run if the data package is not available under the approach we suggest, regular CRAN checks will provide less code coverage than is typical for a CRAN package. Package maintainers should keep this in mind, and they may want to consider alternatives for ensuring regular testing of all examples in the code package. One option may be through use of a regularly scheduled cron job through Travis CI to check the latest stable version of the code package.

It is important to note that, under the suggested approach, proper versioning of any data packages hosted in a drat repository is entirely the responsibility of the owner of that repository. By contrast, $\mathrm{R}$ users who install packages from CRAN can be confident that a version number of a package is tied to a unique version of the source code. While versioning of $R$ data packages can improve research reproducibility (Gentleman et al., 2004), if the owner of the drat repository is not vigilant about changing the version number for every change in the source code of packages posted in the repository, the advantages of packaging the data in terms of facilitating reproducible research are lost. Similarly, the repository owner is solely responsible for archiving older versions of the package, unlike a CRAN package, for which archiving is typically ensured by CRAN. In particular, for very large packages that are updated often, the size limitations of a GitHub repository may force a repository owner to remove older versions from the archive.

\section{Conclusion}

$\mathrm{R}$ packages offer the chance to distribute large datasets while also providing functions for exploring and working with that data. However, data packages often exceed the suggested size of CRAN packages, which is a challenge for package maintainers who would like to share their code through this central and popular repository. Here, we suggest an approach in which the maintainer creates a smaller code package with the code to interact with the data, which can be submitted to CRAN, and a separate data package, which can be hosted by the package maintainer through a personal drat repository. Although drat repositories are not mainstream, and so cannot be listed with an 'Imports' or 'Depends' dependency for a package submitted to CRAN, we suggest a way of including the data package as a suggested package and incorporating conditional code in the executable code within vignettes, examples, and tests, as well as conditioning functions in the code package to check for the availability of the data package. This approach may prove useful for a number of $\mathrm{R}$ package maintainers, especially with the growing trend to the sharing and use of open data in many of the fields in which $\mathrm{R}$ is popular.

\section{Acknowledgements}

We thank Martin Morgan and Roger Peng for comments and suggestion on an earlier draft of this manuscript. This work was supported in part by a grant from the National Institute of Environmental Health Sciences (R00ES022631). 


\section{Bibliography}

Z. W. Almquist. US Census spatial and demographic data in R: The UScensus2000 suite of packages. Journal of Statistical Software, 37(6):1-31, 2010. URL https://doi.org/10.18637/jss.v037.i06. [p487]

B. Anderson, A. Schumacher, W. Crosson, M. Al-Hamdan, M. Yan, J. Ferreri, Z. Chen, S. Quiring, and S. Guikema. hurricaneexposuredata: Data Characterizing Exposure to Hurricanes in United States Counties, 2017a. URL https://github. com/geanders/hurricaneexposuredata. Github repository containing R package. [p487]

B. Anderson, M. Yan, J. Ferreri, W. Crosson, M. Al-Hamdan, A. Schumacher, and D. Eddelbuettel. hurricaneexposure: Explore and Map County-Level Hurricane Exposure in the United States, 2017b. URL https: //CRAN.R-project.org/package=hurricaneexposure. R package version 0.0.1. [p487]

Bioconductor Core Team. Bioconductor- Package guidelines. http: //bioconductor . org/developers / package-guidelines/, 2017. Accessed: 2017-01-31. [p486]

S. Chamberlain, B. Anderson, M. Salmon, A. Erickson, N. Potter, J. Stachelek, A. Simmons, K. Ram, and H. Edmund. Rnoaa: 'NOAA' Weather Data from R, 2016. URL https: //CRAN. R-project.org/ package=rnoaa. R package version 0.6.6. [p486]

G. Csárdi. rcmdcheck: Run 'R CMD Check' from 'R' and Capture Results, 2016. URL https://CRAN. Rproject. org/package $=r c m d c h e c k$. R package version 1.2.1. [p490]

S. Eckel and R. D. Peng. stashR: A Set of Tools for Administering SHared Repositories, 2012. URL https://CRAN.R-project.org/package=stashR. R package version 0.3.5. [p486]

D. Eddelbuettel and J. Horner. littler: $R$ at the Command-Line via ' $r$ ', 2016. URL https://CRAN. Rproject. org/package=littler. R package version 0.3.1. [p490]

D. Eddelbuettel, C. Boettiger, S. Gibb, C. Gillespie, J. Górecki, M. Jones, T. Leeper, S. Pav, and J. Schulz. drat: Drat R Archive Template, 2016. URL https://CRAN. R-project. org/package=drat. R package version 0.1.2. [p487, 488]

R. C. Gentleman, V. J. Carey, D. M. Bates, B. Bolstad, M. Dettling, S. Dudoit, B. Ellis, L. Gautier, Y. Ge, J. Gentry, and others. Bioconductor: Open software development for computational biology and bioinformatics. Genome Biology, 5(10):R80, 2004. URL https://doi .org/10.1186/gb-2004-5-10r80. [p487, 495]

H. Parsonage, T. Cameron, B. Coates, W. Young, and I. Cherastidtham. grattan: Perform Common Quantitative Tasks for Australian Analysts and to Support Grattan Institute Analysis, 2017. URL https: //CRAN.R-project.org/package=grattan. R package version 1.3.0. [p491]

R. D. Peng and F. Dominici. Statistical Methods for Environmental Epidemiology with R: A Case Study in Air Pollution and Health. Springer-Verlag, New York, NY, USA, 2008. [p486]

R Core Team. R: A Language and Environment for Statistical Computing. R Foundation for Statistical Computing, Vienna, Austria, 2017a. URL https: //www.R-project.org/. [p486]

R Core Team. Writing R Extensions. R Foundation for Statistical Computing, Vienna, Austria, 2017b. URL http://CRAN.R-Project.org/doc/manuals/R-exts.html. [p486, 488, 491, 493, 494]

K. Walker and B. Rudis. Tigris: Load Census TIGER/Line Shapefiles into R, 2016. URL https: //CRAN. Rproject. org/package=tigris. R package version 0.3.3. [p486]

H. Wickham. R Packages. O’Reilly Media, Inc., Sebastopol, California, 2015. [p488]

H. Wickham, W. Chang, and RCoreTeam. devtools: Tools to Make Developing R Packages Easier, 2016. URL https://CRAN.R-project. org/package=devtools. R package version 1.12.0. [p490]

Y. Xie. knitr: A General-Purpose Package for Dynamic Report Generation in R, 2016. URL https://CRAN. Rproject. org/package=knitr. R package version 1.15.1. [p494]

G. Brooke Anderson

Colorado State University

Lake Street 
Fort Collins, $\mathrm{CO}$

brooke. anderson@colostate.edu

Dirk Eddelbuettel

Debian and R Projects; Ketchum Trading

Chicago, IL, USA

edd@debian .org 